\title{
8 VIVÊNCIAS DOS ENFERMEIROS FRENTE AO PROCESSO DE MORRER: UMA METASSÍNTESE QUALITATIVA
}

\author{
| Rodrigo Bastos ${ }^{1}$; Fabrício Lamb²; Alberto Quintana ${ }^{3}$; Carmem Beck ${ }^{4}$; Franco Carnevale ${ }^{5}$ |
}

\section{RESUMO}

CONTEXTO: As vivências hospitalares do enfermeiro frente ao processo de morrer têm sido relacionadas à potencialização de sofrimento, especialmente quando o profissional não desenvolve estratégias de enfrentamento, interferindo na assistência prestada.

OBJETIVO: realizar uma metassíntese acerca das vivências hospitalares do Enfermeiro frente à morte e o processo de morrer. MÉTODO: metassíntese realizada por meio da busca na Biblioteca Virtual em Saúde, utilizando os descritores Enfermagem, Atitude frente à morte e Enfermagem de cuidados paliativos na terminalidade da vida. Foram encontrados 170 artigos e selecionados 23 artigos.

RESULTADOS: a maioria dos estressores é advinda da atuação do Enfermeiro junto aos familiares de pacientes. Os fatores facilitadores são subjetivos, relacionados à religiosidade, resistência profissional e comunicação efetiva. Os fatores dificultantes encontrados traziam a tristeza, fracasso, angústia, impotência, culpa, dor ou medo como componentes a serem trabalhados.

CONCLUSÃO: Os fatores facilitadores foram observados como fontes de motivação e suporte para a construção de um processo de trabalho menos desgastante e mais produtivo.

PALAVRAS-CHAVE: Enfermagem; atitude frente à morte; enfermagem de cuidados paliativos na terminalidade da vida

\section{RESUMEN}

"Vivencias de los enfermeros ante el proceso de morir: una meta-síntesis cualitativa"

CONTEXTO: Las experiencias de hospital de las enfermeras contra el proceso de muerte se han relacionado con la potenciación de sufrimiento, sobre todo cuando el profesional no se desarrolla estrategias de supervivencia, lo que interfiere con la asistencia.

OBJETIVO: una meta-síntesis sobre las experiencias de enfermera de hospital que trabaja con el proceso de morir.

MÉTODO: metasíntesis realizado mediante la búsqueda en la Biblioteca Virtual en Salud, utilizando los descriptores: enfermería; actitud frente a la muerte; enfermería de cuidados paliativos al final de la vida. 170 artículos encontrados y seleccionaron 23 artículos publicados.

RESULTADOS: La mayoría de los factores de estrés son procedentes de la relación con las familias de los pacientes. Factores de apoyo son subjetivos, relacionados con la religión, capacidad profesional y comunicación eficaz. Los factores que se encuentran dificultantes trajeron tristeza, el fracaso, la ansiedad, impotencia, culpa, dolor o miedo como componentes para ser trabajados.

CONCLUSIÓN: Los factores predisponentes se han reportado como fuentes de motivación y apoyo para la construcción de un proceso de trabajo sea más sencilla y más productiva.

DESCRIPTORES: Enfermería; actitud frente a la muerte; enfermería de cuidados paliativos al final de la vida.

\begin{abstract}
"The experiences of nurses in the dying process: a qualitative meta-synthesis"

CONTEXT: Hospital experiences of nurses face the process of dying have been related to the potentiation of suffering, especially when the professional does not develop coping strategies, interfering with assistance provided.

OBJECTIVE: To conduct a meta-synthesis about the hospital experiences of nurse face of death and the dying process.

METHOD: meta-synthesis performed through searching the Virtual Health Library, using the descriptors Nursing, Attitude to Death and Hospices and Palliative Care Nursing, written in Portuguese. One hundred and seventy papers were found and twenty-three papers were selected.

RESULTS: Most distressful factors arose from the nurse's work with the families of dying patients. Factors that facilitate the process are subjective, related to religiousness, professional resistance and effective communication. Sadness, failure, anxiety, helplessness, guilt, pain and fear are components to be worked.

CONCLUSION: The predisposing factors have been reported as sources of motivation and support for the construction of a working process less taxing and more productive.
\end{abstract}

\section{KEYWORDS: Nursing; attitude to death; hospice and palliative care nursing}

Submetido em 13-09-2016

Aceite em 30-05-2017

\footnotetext{
1 Enfermeiro. Mestre em Psicologia. Doutorando em Tocoginecologia pela Faculdade de Ciências Médicas da Universidade Estadual de Campinas, almeidabastos.rodrigo@ gmai.com; Endereço: Departamento de Psicologia Médica e Psiquiatria - Prédio FCM11. Cidade Universitária Zeferino Vaz. CEP 13083-887 - Campinas, SP, Brasil. 2 Mestre em Psicologia pela Universidade Federal de Santa Maria, fabriciolamb@hotmail.com; Endereço: Centro de Ciências Sociais e Humanas 3 Doutor em Ciências Sociais e Pós-Doutor na temática de Bioética. Professor Titular da Universidade Federal de Santa Maria, albertom.quintana@gmail.com 4 Enfermeira. Doutora em Enfermagem. Professor Associado IV da Universidade Federal de Santa Maria. Bolsista de Produtividade em Pesquisa do CNPq - Nível 2, carmembeck@gmail.com; Endereço: Centro de Ciências Sociais e Humanas - Prédio 74B, sala 3212a - Av. Roraima nº 1000 Cidade Universitária Bairro Camobi Santa Maria 5 Psicólogo. Doutor em Aconselhamento Psicológico na McGill University; Doutor em Filosofia Moral na Université Laval. Professor Titular da Escola de Enfermagem da McGill University, franco.carnevale@mcgill.ca; Endereço: Ingram School of Nursing - Wilson Hall, 3506 University Street, Room 210. Montreal, Quebec H3A 2 A7.
} 


\section{INTRODUÇÃO}

O significado da morte tem se modificado ao longo dos tempos de acordo com a época, contextos sociais, históricos e culturais. Além disso, os modos de vida de uma sociedade refletem a forma como ela significa a morte (Ariès, 2003).

$\mathrm{Na}$ idade média, a morte possuía um significado de curso natural da vida, o homem possuía capacidade de perceber sinais naturais ou convicções íntimas de sua própria morte, sendo naturalmente aceita (Veras e Moreira, 2012). Havia apenas o medo de não perceber esses sinais e morrer solitário. As vivências profissionais centravam-se em preparar o moribundo e a família para a partida, como se a morte fosse apenas uma mudança de lar (Ariès, 2003; Veras e Moreira, 2012).

A partir do século XVIII, o significado da morte adquire um teor mais dramático, acompanhado por sentimentos saudosistas e dolorosos da perda. As atitudes diante da morte sofrem mudanças e o luto se torna mais exagerado, surgindo sentimentos de negação e de vergonha, tornando-a de interdita ou velada (Carvalho et al., 2006).

Ao longo do século XIX e XX, o hospital passa a ser o local ideal para se morrer, conferindo à morte um significado técnico, no intuito de esvaziar sua carga dramática, caracterizando uma desapropriação da morte e do processo de morrer, onde a morte do sujeito torna-se responsabilidade dos médicos que decidem quando, como e onde morrer (Ariès, 2003; Carvalho et al., 2006).

Ao término da década de 1960, enfermeira inglesa Cecily Saunders inicia a elaboração de estratégias de cuidado integral ao paciente sem possibilidades de cura (Borges e Mendes, 2012). Estes movimentos pró-humanização da morte, promovem reflexões sobre as atitudes e vivências frente à morte e o processo de morrer junto aos profissionais, pacientes e suas famílias, bem como sobre o enfrentamento emocional e questões éticas (Borges e Mendes, 2012; Santos e Bueno, 2010). Entretanto, o processo acadêmico-formativo do profissional de saúde ainda apresenta deficiências quanto ao treinamento adequado para assistir a família e o paciente em seu processo de morrer. No ambiente hospitalar, destaca-se o enfermeiro como o profissional de saúde que permanece mais tempo com o paciente, convivendo direta e periodicamente com o binômio vida/morte e são cobrados a manterem uma postura firme e quase insensível (Santos e Bueno, 2010).
As vivências hospitalares do enfermeiro frente à morte e o processo de morrer têm sido relacionados à potencialização de sofrimento, especialmente quando o profissional não desenvolve estratégias de enfrentamento, interferindo na assistência prestada (Borges e Mendes, 2012).

Do exposto, esse estudo objetiva realizar uma metassíntese acerca das vivências hospitalares do Enfermeiro frente à morte e o processo de morrer. Elaborou-se a seguinte pergunta condutora: Quais estudos estão disponíveis na literatura sobre as vivências hospitalares do Enfermeiro frente à morte e o processo de morrer? Acredita-se que a sumarização dos estudos já publicados sobre a temática subsidiará reflexões e viabilizará suportes e demandas laborais e pessoais do Enfermeiro.

\section{MÉTODO}

Trata-se de uma metassíntese da literatura que é definida como a integração interpretativa de achados qualitativos com metodologias diversas, possibilitando a integração dos achados e oferecendo uma nova interpretação dos resultados (Sandelowski, Docherty \& Emden, 1997). A pesquisa bibliográfica eletrônica foi realizada no ano de 2014, utilizando como fonte de busca as bases de dados indexadas na Biblioteca Virtual da Saúde (BVS).

Utilizou-se a associação dos descritores Enfermagem e Atitude frente à morte em uma primeira busca, e, posteriormente, Enfermagem e Enfermagem de cuidados paliativos na terminalidade da vida.

Os critérios de inclusão contemplaram artigos completos com descritores em português, publicados nos últimos seis anos (entre 2009 e 2014), os quais abordavam as percepções e vivências hospitalares de Enfermeiros frente à morte e o processo de morrer. Os critérios de exclusão descartaram textos científicos que não se caracterizam como artigos originais qualitativos ou com deficiência na descrição metodológica. Os artigos selecionados foram lidos na íntegra, sendo sistematizadas suas principais características. Inicialmente foram construídas três tabelas, sendo que a primeira tabela continha a identificação dos artigos selecionados, a segunda continha os dados relativos aos métodos utilizados em cada pesquisa e a terceira, a análise propriamente dita dos dados obtidos. 
Para compor os resultados desta metassíntese optouse por uma visão integrativa dos resultados, seguindo a sequência metodológica: 1) Metamétodo, onde foi realizada uma análise dos percursos metodológicos adotados em cada estudo e de suas consequências para a perspectiva lançada sobre o problema investigado; 2 ) Meta-análise dos dados, onde foi realizada a análise das análises, confrontando as interpretações realizadas pelos autores, reinterpretando os dados à luz dos achados dos demais estudos que compuseram o universo bibliográfico investigado. Após a descrição dos resultados oriundos da metassíntese, realizou-se a discussão das informações, destacando-se as principais implicações das análises realizadas para o contexto científico e assistencial.

\section{RESULTADOS}

Foram encontrados 119 artigos para os descritores associados Enfermagem e Enfermagem de Cuidados Paliativos na Terminalidade da Vida e 51 artigos para os descritores associados Enfermagem e Atitude Frente à Morte, totalizando 170 artigos. Identificando os artigos que apareciam em mais de uma base de dados, excluiuse 35 artigos da primeira associação de descritores e 15 da segunda. Foram lidos os títulos e os resumos dos 120 artigos restantes, verificando-se o tema e a pergunta condutora. Destes, 46 artigos foram selecionados e 74 foram descartados por não preencherem os critérios de inclusão. Os artigos eliminados, em sua maioria, correspondiam à vivência de auxiliares e técnicos de enfermagem e/ou abordavam o tema na perspectiva de pacientes e familiares.

Após a análise na íntegra quanto à pertinência do tema, foram selecionados 23 artigos como amostra desse estudo, publicados nas revistas: Escola Anna Nery, Ciência \& Saúde Coletiva, Acta Paulista de Enfermagem, Revista Brasileira de Enfermagem, Revista Gaúcha de Enfermagem, Cuidado Fundamental Online, Revista de Eletrônica de Enfermagem, Revista Bioética, Revista Escola de enfermagem USP e Revista Rene.

\section{Metamétodo}

A coleta e análise de dados utilizados dependeram da perspectiva metodológica de cada estudo, dos objetivos e do referencial teórico utilizado por cada autor. $\mathrm{O}$ método de coleta de dados mais frequentemente encontrado nos artigos foi a entrevista semiestruturada (sete) que consiste em um instrumento que possibilita a revelação da fala sob condições estruturais, de sistemas de valores e normas (Minayo, 2010).
Ainda, três estudos associaram a observação participante à entrevista. Outros métodos utilizados foram o da Pesquisa Fenomenológica (quatro), das Representações Sociais (dois), Arco de Juan Charles Magueres (dois), Grupo Focal (dois), pesquisa narrativa (um) e incidente crítico (um).

$\mathrm{Na}$ análise dos dados, nove estudos fizeram uso da Análise de Conteúdo de Bardin, que visa a obtenção sistemática de indicadores que permitam a inferência de conhecimentos relativos às condições de produção das mensagens das falas (Bardin, 2010). O segundo método mais utilizado foi a Análise Temática de Minayo, com cinco estudos, sendo uma técnica da análise de conteúdo que consiste em descobrir os núcleos de sentidos que compõem uma comunicação (Minayo, 2010). Também apareceram as técnicas do Discurso do Sujeito Coletivo (um), Estrutura do Fenômeno Estruturado (um), Pedagogia Problematizadora de Paulo Freire (um), Análise Fenomenológica (dois) e Teoria das Representações Sociais (dois) e um estudo não especificou a metodologia de análise dos dados.

\section{Meta-análise dos dados qualitativos}

Os dados que emergiram foram organizados em dois grupos. O primeiro refere-se às características facilitadoras deste processo e o segundo as dificuldades encontradas por Enfermeiros na sua atuação profissional frente à morte e o processo de morrer.

\section{Fatores que facilitam a atuação dos Enfermeiros}

Os trabalhos analisados demonstram menor observância dos fatores facilitadores do trabalho do Enfermeiro em comparação aos fatores que dificultam. No entanto, sumarizar os aspectos positivos é importante no processo de motivação e suporte ao profissional neste lócus de trabalho. A primeira categoria refere-se à importância da comunicação como fonte promotora de boas relações com a equipe de enfermagem, o paciente e a família.

O humor facilitou a comunicação entre a equipe de trabalho, na forma de riso e comentários nos momentos de integração do grupo de trabalho. Para os instantes de atenção ao paciente, é destacado nos estudos que as habilidades de comunicação abordando o processo de morte são necessárias enquanto instrumento para identificação da fase de luto na qual o paciente está inserido. Dessa forma, consegue-se desenvolver um cuidado integral mais efetivo. Em relação à família, a comunicação se torna fundamental no sentido de manter a boa relação interpessoal. 
O Enfermeiro poderá desenvolver este vínculo em momento de dor e tensão ao permitir, sempre que possível, o contato do paciente com o familiar, bem como informações clínicas precisas e sinceras, promovendo conforto e confiança (Abrão et al., 2013; Pai \& Lautert, 2009; Silva, Campos e Pereira, 2011; Silva e Rocha, 2011).

Outra categoria discutida pelos autores se refere à esperança religiosa dos profissionais nos momentos de perda e de sofrimento assistidos e, por vezes, assumidos como seus. A fé, neste sentido, tem a função de proporcionar sensação de bem-estar ao paciente e familiar, promovendo também a satisfação espiritual do próprio profissional (Moro, Almeida, Rodrigues e Ribeiro, 2010).

A influência religiosa na prática profissional remete à concepção de morte como o fim do sofrimento e princípio do descanso eterno. Nesta dimensão filosófica, não há espaço para pensamentos negativos e o profissional poderá encontrar conforto e ferramentas para reconfortar o moribundo e sua família (Abrão et al., 2013; Moro et al., 2010).

Por fim, alguns artigos trazem como categoria a resiliência devido ao trabalho com a morte. O Enfermeiro passa a ter uma atitude de ressignificação da própria vida, proporcionando crescimento pessoal e profissional (Moro et al., 2010; Silva, Valença e Germano, 2010). Neste processo de resiliência, os profissionais não negavam as dificuldades e o sofrimento em trabalhar com o morrer e os momentos de perda, mas trabalhavam com estas dificuldades para promover sua própria saúde. Trabalhavam com o olhar para a vida, incluindo o processo de morte como natural. Dessa forma, valoriza-se a dignidade do paciente antes e depois de sua morte, gerando, por vezes, satisfação e conforto pelo trabalho realizado (Mota, Gomes, Coelho, Filho e Sousa, 2011; Silva et al., 2010).

Este pensamento de resistência à dor e ao sofrimento, da não negação por parte do profissional, das suas limitações individuais enquanto pessoa favorece um amadurecimento pessoal e espiritual. As falas dos entrevistados, descritas e avaliadas pelos autores dos artigos da amostra dessa revisão, apontam que esta forma de agir e resistir estimula os Enfermeiros a adotar atitudes zelosas. O resultado é o cuidado humanizado e sincero ao moribundo e sua família, bem como a minimização do sofrimento profissional e a maximização do crescimento pessoal (Borges e Mendes, 2012, Mota et al., 2011).

\section{Fatores dificultantes a atuação dos Enfermeiros}

Os relatos dos Enfermeiros contidos nos artigos da amostra dessa revisão mostraram os pedidos incessantes dos familiares para que sejam aplicadas todas as terapias possíveis para retardar a morte dos pacientes, sem perspectiva de melhora, leva a obstinação terapêutica também chamada de distanásia (Silva, Quintana e Nietsche, 2012). Uma das consequências dessa atitude é o receio gerado entre os profissionais em relação às repercussões bioéticas e legais acerca da restrição terapêutica ou suspensão da terapia fútil (Silva et al., 2012; Souza et al., 2013). Isto se reflete na omissão de algumas informações nos prontuários, as quais são passadas verbalmente entre os membros da equipe. Colabora neste sentido a inexistência de leis jurídicas claras, no Brasil, sobre este assunto (Silva et al., 2012).

Outro aspecto importante trazido pelos estudos foi a dificuldade do Enfermeiro em lidar com seus sentimentos relacionados à morte iminente. Desta maneira, evita-se o contato verbal com o paciente, o que pode prejudicar a assistência integral (Fernandes et al., 2013; Silva et al., 2012).

Nestas condições o Enfermeiro é confrontado com a sua própria possibilidade de vivenciar sua finitude, bem como de pessoas próximas (Moro et al. 2010; Peterson \& Carvalho, 2011; Silva et al., 2010; Silva e Rocha, 2011). Ainda, os sentimentos são mais conflituosos quando a morte é de uma criança, gerando até um questionamento do seu papel profissional, visto que esta morte não é concebida como algo natural (Monteiro, Rodrigues e Pacheco, 2012; Silva et al., 2010; Souza et al., 2013).

Alguns mecanismos de defesa foram apontados pelos estudos frente às situações de morte e morrer. A negação, manifestada pela aparente insensibilidade e frieza, foi observada como fator dificultante do amadurecimento pessoal e profissional do Enfermeiro (Mota et al., 2011). Outros mecanismos de defesa relatados foram a assistência baseada exclusivamente na técnica4 e o prolongamento do processo de morrer (Souza et al., 2013). Muitas das dificuldades encontradas pelos Enfermeiros na sua atuação poderiam ter sido evitadas ou ao menos atenuadas por uma melhor formação profissional. Esta é a conclusão que muitos autores chegaram, relatando a falta de oportunidades na graduação para adquirir habilidades, dificuldade de acesso a conhecimentos acerca da temática, de discussões nas instituições de ensino e de espaço para expor o medo, as angústias, as frustrações e a insegurança (Barros et al., 2013; Peterson \& Carvalho, 2011; Santana et al., 2013; Silva e Rocha, 2011; Souza et al., 2013). 
Outro aspecto ligado à formação profissional é o seu direcionamento para a cura, na busca de salvar e prolongar a vida. A morte passa a ser evitada a todo custo, a ser percebida como uma frustração profissional e a não ser aceita (Mota et al., 2011; Santana et al., 2013; Silva et al., 2012), o que gera angústia e medo de que o paciente não possa ser salvo (Borges e Mendes, 2012; Mota et al., 2011; Pai \& Lautert, 2009).

Por fim, foi relatado nos estudos que, para atuar frente a pacientes e familiares que vivenciam o processo de morte e morrer, é necessário uma atuação interdisciplinar. Tal afirmação foi embasada na complexidade do tema o qual envolve dimensões sociais e psicológicas (Waterkemper \& Reibnitz, 2010; Waterkemper, Reibnitz \& Monticelli, 2010). Todos os profissionais da saúde têm sua responsabilidade frente a pacientes e familiares, incluindo o Enfermeiro (Silva, Campos e Pereira, 2011).

\section{DISCUSSÃO}

Embora existam diferentes formas de coleta, análise e interpretação dos dados, os resultados obtidos podem influenciar o caminho do conhecimento e auxiliar na tomada de decisões sobre a temática estudada. Neste estudo fica evidente a relação de estresse frequente entre o Enfermeiro e a família do paciente em situação de terminalidade. O Enfermeiro vê-se numa encruzilhada entre o não uso da obstinação terapêutica e os aspectos legais e éticos. Muitos profissionais optam pelo caminho de atender o desejo do familiar ao invés de trabalhar com ele a compreensão e aceitação da condição do paciente (Silva et al., 2012). Criar um ambiente adequado para dialogar com a família, oportunizar um momento de escuta e formar um vínculo terapêutico pode ajudar na compreensão do estado de saúde do familiar e na tomada de decisões, minimizando a ocorrência de obstinação terapêutica.

Oportunizar um espaço de ajuda para que os profissionais entrem em contato com seus sentimentos e possam trabalha-los é essencial, evitando a utilização de mecanismos de defesa, afastamento físico e emocional do paciente em situação de terminalidade (Peterson \& Carvalho, 2011). Não negar o processo de morte contribui para que o Enfermeiro possa pensar o uso adequado de tecnologias e terapêuticas, bem como evitar o sofrimento desnecessário e o prolongamento do processo de morrer. A morte precisa voltar a ser vista como algo natural e não como fracasso profissional sempre envolto em sentimentos de impotência e frustração.
A morte, enquanto fracasso profissional, está atrelada à formação profissional voltada para a cura e negligente na discussão acerca da terminalidade da vida na formação acadêmica. A vivência de sentimentos negativos relaciona-se à formação inadequada em preparar os profissionais para lidar com o sofrimento e a morte e a ênfase dada apenas na recuperação da vida. Trazer para as salas de aula da graduação as questões vinculadas à morte e ao processo de morrer é essencial para que os futuros profissionais possam intervir junto aos pacientes e familiares de maneira holística e com menos sofrimento (Silva e Rocha, 2011).

Fortalecer a formação dos Enfermeiros pode refletir na satisfação no trabalho e na qualidade da assistência, possibilitando a compreensão das vivências enfrentadas pelos pacientes e familiares. A Sistematização da Assistência de Enfermagem é uma importante estratégia de gestão do cuidado para atender as demandas e necessidades de saúde do paciente e familiar (Silva e Moreira, 2011).

As vivências ligadas à morte são sempre multifacetadas, envolvendo diversos aspectos familiares, sociais, psicológicos e, para tanto, necessitam de atuação interdisciplinar. Quanto aos cuidados paliativos e na terminalidade da vida, um dos fatores importantes frente ao alívio da dor emocional e física é a presença de uma equipe multiprofissional (Waterkemper et al., 2010). Por melhor que seja a atuação do Enfermeiro, o trabalho em equipe, o apoio entre os pares e a presença de outros profissionais de diferentes áreas de conhecimento mostra-se essencial na qualidade da assistência prestada.

Observou-se, neste estudo, que os fatores facilitadores são subjetivos, necessitando de análises conceituais pouco evidenciadas na literatura pesquisada, relacionadas à religiosidade. Aspectos como a resistência profissional diante das vivências com a morte e a comunicação efetiva com a equipe e com o binômio paciente-família, são problematizados na literatura, ganhando destaque na discussão deste estudo. Do ponto de vista teórico, a comunicação efetiva é recomendada para construção de vínculo em quaisquer planos de cuidado, para que a assistência seja realizada com caráter humanitário e de qualidade (Haddad, Machado, Neves-Amado \& Zoboli, 2011; Stefanelli, Carvalho e Arantes, 2005). No caso do trabalho dos Enfermeiros com a morte, a tensão entre o profissional e o binômio paciente-família é um fator complicador, tornando o profissional, por vezes, alheio ao sofrimento e prioritariamente tecnicista. 
Ao analisar, neste artigo de revisão, os autores que trabalham com a temática da resiliência profissional, percebe-se que a resistência observada nos Enfermeiros tem um significado além do ser positivo ou ser negativo. Uma resistência que parte de relações em que não se nega o sofrimento, mas se reflete sobre ele, tornandoo fonte de crescimento pessoal. Este tipo de resistência é descrito por Michel Foucault como práticas de liberdade (Foucault, 2014; Foucault, 2012). Estas práticas são ferramentas fundamentais para a construção do cuidado de si. Implica na estruturação de uma verdade individual, que permitirá ao indivíduo observar e reagir às relações de poder. Esta relação com a verdade é denominada Estética da Existência, na qual o sujeito tem uma relação sólida consigo mesmo, tomando decisões próprias, baseadas em uma postura ético-reflexiva diante de situações adversas (Foucault, 2012).

O processo de cuidar se integra perfeitamente neste contexto, no qual o Enfermeiro assume o papel de líder na resistência natural da equipe às vivências com os processos de morte e morrer.

\section{CONSIDERAÇÕES FINAIS}

Este estudo permitiu a descrição e discussão dos fatores dificultantes e facilitadores do trabalho do Enfermeiro com a morte, baseado em uma revisão da literatura sobre o tema. Observou-se um predomínio de fatores estressores e manifestações de sofrimento por parte dos enfermeiros diante das tensões naturalmente existentes no trabalho com sujeitos sem possibilidades de cura. No entanto, os fatores facilitadores foram encontrados e podem ser fontes de motivação para a construção de um processo de trabalho menos desgastante e mais produtivo.

Pôde-se observar que um aspecto importante trazido pelos estudos foi a dificuldade do Enfermeiro, enquanto ser humano, de lidar com seus sentimentos. Relatados pelos autores nas falas dos profissionais, a tristeza, fracasso, angústia, impotência, insegurança, culpa, dor ou medo são componentes a serem trabalhados. A maturidade do profissional é desenvolvida a partir do processo de gerenciamento destes componentes, tornando-o apto, então, a lidar melhor com os sentimentos de pacientes e familiares, bem como com seus próprios sentimentos. Os fatores que facilitam ou motivam o trabalho do Enfermeiro com a morte não exclui a presença dos estressores. Ao contrário, o gerenciamento eficaz destes fatores é o propulsor de um ambiente de trabalho saudável e produtivo.
Comunicar-se, criar vínculos e, ainda, desenvolver resistência às situações problema, são formas, não apenas de proteger suas emoções, mas de agir em prol de um autorreconhecimento.

A sumarização da literatura disponível acerca da morte e o processo de morrer, vivenciados por enfermeiros hospitalares, colabora para a acessibilidade do conhecimento aos enfermeiros assistenciais, que muitas vezes, devido à sobrecarga de trabalho, não conseguem se aproximar de especializações formais e acadêmicas. A partir desta realidade, este levantamento bibliográfico possibilitou a discussão de suportes motivacionais e produtivos para os Enfermeiros, os quais abarcam esta demanda. Traduziu-se, ainda, em um alerta sobre as barreiras que estes profissionais deverão gerenciar nos momentos de tensão e resistência, naturalmente presentes frente aos leitos de morte.

\section{REFERÊNCIAS BIBLIOGRÁFICAS}

Abrão, F.M.S., Góis, A.R.S., Souza, M.S.B., Araujo, R.A., Cartaxo, C.M.B., \& Oliveira, D.C. (2013). Representações sociais de enfermeiros sobre a religiosidade ao cuidar de pacientes em processo de morte. Revista Brasileira de Enfermagem, 66(5), 730-737.

Ariès, P. (2003). História da morte no ocidente. Rio de Janeiro: Ediouro.

Bardin L. (2010). Análise de conteúdo. Lisboa: Edições 70.

Barros, N.C.B., Alves, E.R.P., Oliveira, C.D.B., Dias, M.D., França, I.S.X., \& Freire, M.E.M. (2013). Cuidados paliativos na uti: compreensão dos enfermeiros. Revista de Pesquisa: Cuidado é Fundamental (Online). 5(1), 3293-3301.

Borges, M.S., \& Mendes, N. (2012). Representações de profissionais de saúde sobre a morte e o processo de morrer. Revista Brasileira de Enfermagem, 65(2), 324-331.

Carvalho, L., Silva, C., Oliveira Santos, A., Oliveira, M., Portela, S., \& Regebe, C. (2006). Perception of the death and dying of the nursing students. A qualitative study. Online Brazilian Journal Of Nursing, 5(3). doi:10.5935/1676-4285.2006507

Fernandes, M.A., Evangelista, C.B., Platel, I.C.S., Agra, G., Lopes, M.S., \& Rodrigues, F.A. (2013). Percepção dos enfermeiros sobre o significado dos cuidados paliativos em pacientes com câncer terminal. Ciência \& Saúde Coletiva. 18(9), 2589-2596. 
Foucault, M. (2012). A ética do cuidado de si como prática da liberdade. In: M. Foucault (Org.) Ditos \& Escritos V - Ética Sexualidade Política (pp. 264-287). Rio de Janeiro: Forense Universitária.

Foucault, M. (2014). História da Sexualidade III: o cuidado de si. Rio de Janeiro: Graal.

Haddad, J.G.V., Machado, E.P., Neves-Amado, J., \& Zoboli, E.L.C.P. (2011). A comunicação terapêutica na relação enfermeiro-usuário da atenção básica: um instrumento para a promoção da saúde e cidadania. $\mathrm{O}$ Mundo da Saúde. 35(2), 145-155.

Lopes, A.L.M., \& Fracolli, L.A. (2008). Revisão sistemática de literatura e metassíntese qualitativa: considerações sobre sua aplicação na pesquisa em enfermagem. Revista Texto \& Contexto Enfermagem. 17(4), 771-8.

Minayo, M.C. (2010). O desafio do Conhecimento: pesquisa qualitativa em saúde. São Paulo: Hucitec.

Monteiro, A.C.M., Rodrigues, B.M.R.D., \& Pacheco, S.T.A. (2012). O enfermeiro e o cuidar da criança com câncer sem possibilidade de cura atual. Escola Anna Nery. 16(4), 741-746.

Moro, C.R., Almeida, I.S., Rodrigues, B.M.R.D. \& Ribeiro, I.B. (2010). Desvelando o processo de morrer na adolescência: a ótica da equipe de enfermagem. Revista Rene. 11(1), 48-57.

Mota, M.S., Gomes, G.C., Coelho, M.F., Filho, W.D.L., \& Sousa, L.D. (2011). Reações e sentimentos de profissionais da enfermagem frente à morte dos pacientes sob seus cuidados. Revista Gaúcha de Enfermagem. 32( 1 ), 129-135.

Pai, D.D., \& Lautert, L. (2009). Estratégias de enfrentamento do adoecimento: um estudo sobre o trabalho da enfermagem. Acta Paulista de Enfermagem. 22( 1), 60-5.

Peterson, A. A., \& Carvalho, E. C. (2011). Comunicação terapêutica na enfermagem: dificuldades para o cuidar de idosos com câncer. Revista Brasileira de Enfermagem, 64(4), 692-697.

Sandelowski, M., Docherty, S. \& Emden, C. (1997). Focus on qualitative methods. Qualitative metasynthesis: issues and techniques. Research in Nursing \& Health. 20(4), 365-71.

Santana, J., Santos, A., Silva, B., Oliveira, D., Caminha, E., Peres, F., Andrade, C., \& Viana, M. (2013). Docentes de enfermagem e terminalidade em condições dignas. Revista Bioética, 21(2) 298-307.
Santos, J.L. \& Bueno, S.M.V. (2010). A questão da morte e os profissionais de enfermagem. Revista Enfermagem UERJ. 18(3), 484-7.

Silva, C.A.X., Morais, F.R.R., Oliveira, L.C., Queiroz, J.C., Soares, F.R.R., \& Carvalho, F.P.B. (2012). Cuidados paliativos: uma alternativa para os usuários oncológicos fora de possibilidades terapêuticas. Revista de Pesquisa: Cuidado é Fundamental (Online). 4(4), 2797-2804.

Silva, K.C.O., Quintana, A.M., \& Nietsche, E.A. (2012). Obstinação terapêutica em unidade de terapia intensiva: perspectiva de médicos e enfermeiros. Escola Anna Nery. 16(4), 697-703.

Silva, L.C.S.P., Valença, C.N., \& Germano, R.M. (2010). Percepções dos profissionais de enfermagem intensiva frente a morte do recém-nascido. Revista Brasileira de Enfermagem. 63(2), 238-242.

Silva, M.K.G., \& Rocha, S.S. (2011). O significado de cuidar do recém-nascido sem possibilidade de terapêutica curativa. Revista Rene. 12 (1), 97-103.

Silva, M.M., \& Moreira, M.C. (2011). Sistematização da assistência de enfermagem em cuidados paliativos na oncologia: visão dos enfermeiros. Acta Paulista de Enfermagem. 24(2), 172-178.

Silva, R.S., Campos, A.E.R., \& Pereira, A. (2011). Cuidando do paciente no processo de morte na Unidade de Terapia Intensiva. Revista Escola de enfermagem USP. 45( 3 ), 738-44.

Souza, L.F., Misko, M.D., Silva, L., Poles, K., Santos, M.R., \& Bousso, R.S. (2013). Dignified death for children: perceptions of nurses from an oncology unit. Revista Escola de enfermagem USP. 47(1), 30-37.

Stefanelli, M.C., Carvalho, E.C., \& Arantes, E.C. (2005). Comunicação e enfermagem. In: M.C. Stefanelli \& E.C. Carvalho (Org.), A comunicação nos diferentes contextos da enfermagem (pp. 1-8). Barueri, SP: Manole.

Veras, L., \& Moreira, V. (2012). A morte na visão do sertanejo nordestino em tratamento oncológico. Estudos de Psicologia (Natal), 17(2), 291-298.

Waterkemper, R., \& Reibnitz, K.S. (2010). Cuidados paliativos: a avaliação da dor na percepção de enfermeiras. Revista Gaúcha de Enfermagem. 31(1), 84-91.

Waterkemper, R., Reibnitz, K.S., \& Monticelli, M. (2010). Dialogando com enfermeiras sobre a avaliação da dor oncológica do paciente sob cuidados paliativos. Revista Brasileira de Enfermagem. 63(2), 334-339. 